\title{
WEIGHTED REPRESENTATIONS OF A PRIMITIVE ALGEBRA
}

\author{
E. G. GOODAIRE ${ }^{1}$
}

\begin{abstract}
Let $L$ be a diagonable subspace of an associative algebra $A$ with identity over a field $F$; that is, $L$ is spanned by a set of pairwise commuting elements, and the linear transformations ad $x: a \mapsto a x-x a$ for $x \in L$ are simultaneously diagonalizable. Denote the centralizer of $L$ in $A$ by $\mathscr{C}$. A module $V$ over $A$ or $\mathscr{C}$ is $L$-weighted if for some nonzero $v \in V$ and map $\lambda: L \rightarrow F, v(x-\lambda(x) 1)^{n(x)}=0$ for each $x \in L$, and $x$-weighted if for some nonzero $v \in V, \lambda \in F$ and positive integer $n, v(x-\lambda 1)^{n}=0$. In this paper we give conditions under which the following statements are equivalent:

1. All irreducible modules over $A$ and $\mathscr{C}$ are $L$-weighted.

2. For each $x \in L$, some irreducible $A$-module is $x$-weighted and $x$ is algebraic over $F$.
\end{abstract}

Let $A$ be an associative algebra with 1 over a field $F$ of characteristic 0 which possesses a diagonable subspace. By this, we mean a subspace $L$ spanned by a set of pairwise commuting elements such that $\{\operatorname{ad} x: x \in L\}$ is a simultaneously diagonalizable set of linear transformations of $A$, where for any $x, a \in A$, ad $x: a \mapsto(a, x) \equiv a x-x a$. Corresponding to $L$, there is a collection $\Delta$ of maps $\alpha: L \rightarrow F$, called roots of $L$ in $A$, such that $A$ decomposes into $\bigoplus \sum_{\alpha \in \Delta} A_{\alpha}$, where $A_{\alpha}=A_{\alpha}(L)=\{a \in A:(a, x)=\alpha(x) a$ for every $x \in L\}$. A module $V$ over any subalgebra of $A$ containing $L$ is said to be $L$-weighted if there is a map $\lambda: L \rightarrow F$, called a weight of $L$ in $V$, and a nonzero $v \in V$, such that $v(x-\lambda(x) 1)^{n}=0$ for $n=n(x) \in N$, the natural numbers, and each $x \in L$. If for some $x \in L, n \in N$, and $\lambda \in F$, $v(x-\lambda 1)^{n}=0$, then $V$ is $x$-weighted. In fact, any weight or root is always a linear functional on $L$ [1]. It is known [1] that should $V$ be both $L$ weighted and irreducible, then $V$ has a weight space decomposition, $V=\bigoplus \sum_{\lambda \in \Lambda} V_{\lambda}$ relative to the complete set $\Lambda$ of weights of $L$ in $V$. Here $V_{\lambda}=\left\{v \in V: v(x-\lambda(x) 1)^{n(x)}=0\right.$ for each $\left.x \in L\right\}$ is the weight space corresponding to $\lambda$.

Received by the editors April 11, 1973.

AMS (MOS) subject classifications (1970). Primary 16A12, 16A20; Secondary $17 \mathrm{~B} 10$.

Key words and phrases. Diagonable subspace, weighted representation.

1 Part of this work appears in the author's doctoral dissertation written at the University of British Columbia under the supervision of Dr. C. T. Anderson.

(c) American Mathematical Society 1974 
Now $A_{0}(L)$ is a subalgebra of $A$ containing 1 and $L$, for it is just the centralizer $\mathscr{C}$ of $L$ in $A$. This centralizer plays an important role in the representation theory for $A$ : we have shown elsewhere that a theorem of Lemire [2] concerning the weighted irreducible representations of a finitedimensional Lie algebra over an algebraically closed field of characteristic 0 is a consequence of the fact that any Cartan subalgebra of such a Lie algebra is a diagonable subspace of the universal enveloping algebra. For a fixed map $\lambda: L \rightarrow F$, the irreducible $L$-weighted representations of $A$ which have $\lambda$ as a weight are in one-to-one correspondence with the same class of representations of $\mathscr{C}$, up to equivalence [1].

Now the mere existence of a diagonable subspace in $A$ does not guarantee that all irreducible $A$-modules are weighted, as an example in [3] shows; furthermore, it is not clear that the existence of weight space decompositions for all irreducible $A$-modules implies such decompositions for all irreducible $\mathscr{C}$-modules. In this paper, we give a necessary and sufficient condition for all the representations of $A$ and $\mathscr{C}$ to be $L$-weighted. The notation and conventions of our first paragraph will be everywhere employed.

THEOREM. Let $A=\bigoplus \sum_{\alpha \in \Delta} A_{\alpha}$ be the decomposition of a primitive algebra $A$ corresponding to a diagonable subspace $L$ with centralizer $\mathscr{C}$. Suppose $\Delta$ is finite and $L$ is finite dimensional. Then the following are equivalent:

(1) All irreducible modules of $A$ and $\mathscr{C}$ are $L$-weighted.

(2) For each $x \in L, x$ is algebraic and some irreducible A-module is $x$-weighted.

EXAMPLE. We begin with an example intended to show the complexity of the problem of determining which representations of an algebra are weighted. Let $A$ be the universal enveloping algebra of the two-dimensional nonabelian Lie algebra over a field $F$ (of characteristic 0 ). Then $A$ is just the ring of noncommuting polynomials over $F$ in two indeterminates $x$ and $y$, with $y x=(x+1) y$. One can verify that, in fact, $y^{i} f(x)=f(x+i 1) y^{i}$ for any $i \in N$ and $f(x) \in F[x]$. The subspace $F x$ is diagonable and $A=\bigoplus \sum_{\alpha_{n} \in \Delta} A_{\alpha_{n}}$ where $\alpha_{n}: x \mapsto n x$ for any integer $n \geqq 0$. In fact, $A_{\alpha_{n}}$ is merely $F[x] y^{n}$. We prove first that $A$ is primitive. To this end, note that $y+1$ is not invertible in $A$ and so must be contained in a maximal right ideal $J$ which has the property

$$
f(x) y^{k} \in J \text { for } k>0 \text {, and } f(x) \in F[x] \text { implies } f \equiv 0 .
$$

This follows by induction on $n=\operatorname{degree} f$ and $k$. For $n=0$, if $\alpha y^{k}$ and $y+1$ are both in $J$, then $J$ contains 1 because these are relatively prime polynomials. Assuming the validity of (1) for polynomials $f$ with degree 
less than $n$, suppose $f(x) \in F[x]$ has degree $n$ and $f(x) y^{k}$ and $y+1$ are both in $J$. Then $J$ must contain

$$
\left(f(x) y^{k}, y+1\right)=f(x) y^{k+1}-y f(x) y^{k}=(f(x)-f(x+1)) y^{k+1} .
$$

Since $f(x)-f(x+1)$ has degree less than $n, f(x)=f(x+1)$ by induction. This means $\xi+m$ is a root of $f$ for every integer $m \geqq 0$ and any root $\xi$ of $f$. But this is impossible unless $f$ has degree 0 , and this possibility has already been eliminated. We therefore have (1). It follows immediately that $J$ can contain no two-sided ideal of $A$ except (0) because any such ideal $T$ can be written $T=\bigoplus \sum_{\alpha_{n} \in \Delta} T \cap A_{\alpha_{n}}$ ( $\S 4$ of [1]). Hence $A / J$ is a simple, faithful $A$-module and $A$ is primitive.

Now $A / J$ is not weighted, for we can prove

$$
\left(\sum_{i=0}^{n} a_{i}(x) y^{i}\right)(x-\alpha 1)^{m} \in J \text { implies } \sum_{i=0}^{n} a_{i}(x) y^{i} \in J
$$

where $a_{i}(x) \in F[x], i=0, \cdots, n$. For $n=0, a_{0}(x)(x-\alpha 1)^{m} \in J$ implies $a_{0}(x)=0$ or $x=\alpha 1$ by (1), and the latter possibility is false. If $J$ contains

$$
\left(\sum_{i=0}^{n} a_{i}(x) y^{i}\right)(x-\alpha 1)^{m}=\sum_{i=0}^{n} y^{i} a_{i}(x-i 1)(x-\alpha 1)^{m}
$$

then it also contains

$$
(y+1)^{n} b(x)(x-\alpha 1)^{m}=\sum_{i=0}^{n}\left(\begin{array}{l}
n \\
i
\end{array}\right) y^{i} b(x)(x-\alpha 1)^{m}, \quad b(x)=a_{n}(x-n 1)
$$

because $y+1 \in J$, and so $J$ contains the difference of these two elements; namely,

$$
\begin{aligned}
\sum_{i=0}^{n-1} y^{i}\left(a_{i}(x-\right. & \left.i 1)-\left(\begin{array}{l}
n \\
i
\end{array}\right) b(x)\right)(x-\alpha 1)^{m} \\
& =\sum_{i=0}^{n-1}\left(a_{i}(x)-\left(\begin{array}{l}
n \\
i
\end{array}\right) b(x+i 1)\right) y^{i}(x-\alpha 1)^{m} .
\end{aligned}
$$

By induction, we may assume $a_{i}(x)=\left(\begin{array}{l}n \\ i\end{array}\right) b(x+i 1)$; i.e. that $a_{i}(x)=$ $\left(\begin{array}{l}n \\ i\end{array}\right) a_{0}(x+i 1)$, for $i=0, \cdots, n-1$. Thus

$$
\sum_{i=0}^{n} a_{i}(x) y^{i}=\sum_{i=0}^{n}\left(\begin{array}{l}
n \\
i
\end{array}\right) a_{0}(x+i 1) y^{i}=\sum_{i=0}^{n}\left(\begin{array}{l}
n \\
i
\end{array}\right) y^{i} a_{0}(x)=(1+y)^{n} a_{0}(x)
$$

is in $J$. Hence we have ( 2 ) and $A / J$ cannot be weighted.

On the other hand, some $A$-modules are weighted; for example, there is a maximal right ideal $I$ of $A$ containing $x$ (because $x$ is not invertible) and so $A / I$ is an irreducible $F x$-weighted $A$-module $((I+1) \propto x=0$ for any $\alpha \in F$ ). 
Proposition. If $L$ is a finite-dimensional diagonable subspace of $A$ and $V$ is an irreducible A-module, then $V$ is $L$-weighted if and only if $V$ is $x$-weighted for every $x \in L$.

Proof. If $V$ is $L$-weighted, then $V$ is $x$-weighted for every $x \in L$ by definition. Conversely, suppose $x_{1}, \cdots, x_{k}$ is a basis for $L$. By induction, we assume that there exist scalars $\lambda_{1}, \cdots, \lambda_{k-1}$, and positive integers $n_{1}, \cdots, n_{k-1}$, such that $v\left(x_{i}-\lambda_{i} 1\right)^{n_{i}}=0$ for some nonzero $v \in V, i=1, \cdots$, $k-1$. Now, because $V$ is $x_{k}$-weighted, it is easy to see that $V$ has a weight space decomposition relative to the diagonable subspace $F x_{k}, V=\bigoplus \sum_{\gamma \in \Gamma} V_{\gamma}$, and so we can write $v=\sum v_{\gamma}, v_{\gamma} \in V_{\gamma}$. Using the fact that $V_{\gamma} A_{0}\left(F x_{k}\right) \subset V_{\gamma}$ (see [1]), $v\left(x_{i}-\lambda_{i} 1\right)^{n_{i}}=0, i=1, \cdots, k-1$, implies $v_{\gamma}\left(x_{i}-\lambda_{i} 1\right)^{n_{i}}=0$, $i=1, \cdots, k-1$. Clearly for some $\gamma, v_{\gamma} \neq 0$, so defining $u=v_{\gamma}$ and $\lambda_{n}=\gamma\left(x_{k}\right)$ we have $u\left(x_{i}-\lambda_{i} 1\right)^{n_{i}}=0$ for $i=1, \cdots, k$. Define $\lambda: L \rightarrow F$ by $\lambda\left(x_{i}\right)=\lambda_{i}$ and extend to $L$ by linearity. Then a straightforward calculation shows that $u(x-\lambda(x) 1)^{M}=0, M=\sum_{i=1}^{k} n_{i}$, for any $x \in L$. Thus $V$ is $L$-weighted.

LEMMA 1. Let $L$ be a diagonable subspace of a prime algebra $A$ over $F$ which possesses only a finite set $\Delta$ of roots. Suppose $x \in L$ is algebraic and $X$ is any subalgebra of $A$ containing $x$. Then if any irreducible $X$-module is $x$-weighted, every irreducible $X$-module is $x$-weighted.

PROOF. It is sufficient to prove that the minimal (monic) polynomial $p(t)$ over $F$ which $x$ satisfies has all its roots in $F$. The key step towards this is establishing that $p(t)$ has the form

$$
p(t)=\prod_{\alpha \in S} q(t+\alpha)
$$

where $q(t) \in F[t]$ is irreducible and $S \subset \mathfrak{U}=\{\alpha(x): \alpha$ a root of $F x$ in $A\}$. Since $A$ is semiprime, so is the centralizer $A_{0}(x)$ of $x$ by [1]. Thus $p(t)$ must be of the form $p_{1}(t) \cdots p_{s}(t)$, where $p_{1}(t), \cdots, p_{s}(t)$ are the distinct monic irreducible factors of $p(t)$ in $F[t]$. Let $A_{i j}$ denote the subspace $\left\{a \in A: p_{i}(x) a=0=a p_{j}(x)\right\}$ for $1 \leqq i, j \leqq s$. Then we have

$$
A=\bigoplus \sum_{i, j=1}^{s} A_{i j}
$$

To prove this, we note that the linear transformation $L_{x}: a \mapsto x a$ of $A$ is algebraic with minimal polynomial also $p(t)$ (all algebras we consider contain 1). Thus $A=\bigoplus \sum_{i=1}^{s} A_{i}$ where $A_{i}=\left\{a \in A: p_{i}(x) a=0\right\}$. Now each subspace $A_{i}$ is invariant under the linear transformation $R_{x}: a \mapsto a x$, which is also algebraic with minimal polynomial $p(t)$. Thus the restriction 
of $R_{x}$ to $A_{i}$ has minimal polynomial dividing $p(t)$, and so each $A_{i}$ decomposes into a direct sum of spaces $A_{i j}$ for some integers $j, 1 \leqq j \leqq s$. This gives (4).

Now for each $k, 1 \leqq k \leqq s$, define $a_{k}=\prod_{i \neq k} p_{i}(x)$. Then $a_{k} \neq 0$, and for any $i, j \in\{1, \cdots, s\}, a_{i} A a_{j} \neq 0$ because $A$ is prime. But $a_{i} A a_{j} \subset A_{i j}$ and so each of the spaces $A_{i j}$ is nonzero. Suppose then that $0 \neq a \in A_{j 1}$ and $a=\sum_{\alpha \in \mathscr{U}} a_{\alpha}$ is the decomposition of $a$ relative to $F x$ (i.e. $A=\bigoplus \sum_{\alpha \in \mathfrak{X}} A_{\alpha}(x)$ ). For some $\alpha, a_{\alpha} \neq 0$, and because $A_{\alpha}(x) A_{0}(x) \subset A_{\alpha}(x)$ and the sum $\sum_{\alpha \in \vartheta} A_{\alpha}(x)$ is direct, $a_{\alpha} p_{1}(x)=0$. But $\left(a_{\alpha}, x\right)=\alpha a_{\alpha}$ easily implies $p_{1}(x+\alpha) a_{\alpha}=0$. We have also $p_{j}(x) a=0$ and so just as above $p_{j}(x) a_{\alpha}=0$. Thus the polynomials $p_{1}(t+\alpha)$ and $p_{j}(t)$ cannot be relatively prime, and because they are irreducible and monic, $p_{j}(t)=p_{1}(t+\alpha)$. This establishes (3), where $q(t)=p_{1}(t)$.

Now by hypothesis, some irreducible $X$-module $V$ is $x$-weighted, and the existence of a nonzero $v \in V$ and $\lambda \in F$ with $v(x-\lambda 1)^{n}=0$ certainly implies $(x-\lambda 1)$ is not invertible in $X$. Thus the polynomials $p(t)$ and $t-\lambda$ are not relatively prime; i.e. $t-\lambda$ divides $p(t)$. It follows that for some $\alpha \in S, q(t+\alpha)=t-\lambda$; hence $q(t)=t-(\lambda+\alpha)$ and all roots of $p(t)$ lie in $F$.

This lemma, together with the proposition, gives one half of our theorem (because primitive algebras are prime). The other half is valid for $L$ of arbitrary dimension.

LEMMA 2. Let $A$ be a primitive algebra over $F$ which decomposes $A=\bigoplus \sum_{\alpha \in \Delta} A_{\alpha}$ relative to a diagonable subspace $L$. Then if $\Delta$ is finite and all irreducible modules over $A$ and $\mathscr{C}$ (the centralizer of $L$ ) are $L$ weighted, all elements of $L$ are algebraic over $F$.

Proof. We make use of Theorem 1.6 in [1] which states that $\mathscr{C}$ is a direct sum of finitely many primitive algebras $R_{i}, i=1, \cdots, n$. If $I$ is any maximal right ideal of $\mathscr{C}, \mathscr{C} \mid I$ is an irreducible $\mathscr{C}$-module and hence weighted; i.e. for some $\lambda: L \rightarrow F$ and $u \in \mathscr{C} \backslash I, u(x-\lambda(x) 1)^{n(x)} \in I$ for every $x \in L$. Since $x-\lambda(x) 1$ is in the centre of $\mathscr{C}$, it follows that $x-\lambda(x) 1 \in I$. For each $i$, there exists a maximal right ideal $J_{i}$ of $R_{i}$ containing no nonzero ideal of $R_{i}$. The above argument then shows that $J_{i} \oplus \sum_{j \neq i} R_{j}$, which is a maximal right ideal of $\mathscr{C}$, contains $x-\lambda_{i}(x) 1$ for every $x \in L$ and some $\lambda_{i}: L \rightarrow F, i=1, \cdots, n$. For a particular $x \in L$, write $x=\sum_{i=1}^{n} x_{i}, x_{i} \in R_{i}$, and $1=\sum_{i=1}^{n} e_{i}, e_{i}$ the identity of $R_{i}$. By looking at the $i$-component of $x-\lambda_{i}(x) 1$, we see that $x_{i}-\lambda_{i} e_{i} \in J_{i}, \lambda_{i}=\lambda_{i}(x)$. But $x_{i}-\lambda_{i} e_{i}$ is in the centre of $R_{i}$ because $x-\lambda(x) 1$ is in the centre of $\mathscr{C}$. Thus $\left(x_{i}-\lambda_{i} e_{i}\right) R_{i}$ is an ideal of $R_{i}$ contained in $J_{i}$. It now follows that $x_{i}=\lambda_{i} e_{i}$, and $x=\sum_{i=1}^{n} \lambda_{i} e_{i}$ with $e_{1}, \cdots, e_{n}$ pairwise orthogonal idempotents. Since $x$ satisfies the polynomial $\prod_{i=1}^{n}\left(t-\lambda_{i}\right) \in F[t]$, it is algebraic. 


\section{REFERENCES}

1. E. G. Goodaire, Irreducible representations of algebras, Canad. J. Math. (to appear).

2. F. W. Lemire, Weight spaces and irreducible representations of simple Lie algebras, Proc. Amer. Math. Soc. 22 (1969), 192-197. MR 39 \#4326.

3. - Existence of weight space decompositions for irreducible representations of simple Lie algebras, Canad. Math. Bull. 14 (1971), 113-115. MR 45 \#328.

Department of Mathematics, University of British Columbia, Vancouver, British Columbia, Canada

Current address: Department of Mathematics, Memorial University, St John's, Newfoundland, Canada 